Case Report

\title{
Post streptococcal reactive arthritis in children: varied presentations, differentiation from acute rheumatic fever and antibiotic prophylaxis
}

\author{
Swaminathan A. ${ }^{1}$, Muhammad N. ${ }^{2}$, Benjamin S. ${ }^{3}$, Rajagopalan K.S. ${ }^{4}$ \\ ${ }^{1}$ Dr. Aravind Swaminathan, Assistant Professor of Paediatrics, Saveetha Medical College and Hospital, Chennai, \\ ${ }^{2}$ Dr. Muhammad Najih, CICU, Great Ormond Street Hospital, London, UK, ${ }^{3}$ Dr. Benjamin Sagayaraj, Professor, \\ ${ }^{4}$ Dr.Kamalnath Sankaran Rajagopalan, Intern, both are affiliated with Department of Paediatrics, Saveetha \\ Medical College and Hospital, Chennai, Tamil Nadu, India.
}

Address for Correspondence: Dr. Aravind Swaminathan (Paed), Assistant Professor, Department of Paediatrics, Saveetha Medical Collegeand Hospital, Thandalam, Kancheepuram, Tamil Nadu, India. Email: draravind08@gmail.com

\begin{abstract}
Post streptococcal reactive arthritis (PSRA) refers to inflammation of one or more joints soon after streptococcal throat infection without a demonstrable presence of the organisms in the joint. We present here, two children with very different forms of PSRA. While the first child had axial and appendicular joint involvement in non-migratory pattern that persisted for a long time with intermittent exacer bation without carditis and with no preceding clinically apparent throat infection,the second child presented with throat infections followed by involvement of knee joints alone in episodic and migratory patterns with disease free intervals in between, but with evidence of mild carditis. Clinical suspicion and close attention to the differentiating features from acute rheumatic fever (ARF) are necessary to arrive at the appropriate diagnosis which could at times be challenging. The need for long term follow up is emphasized as the former child was noted to evolve into enthesitis related arthritis. Presented is a review of differentiating features between ARF and PSRA and the role of antibiotic prophylaxis.
\end{abstract}

Key words- Arthritis, post infectious, Antibiotic prophylaxis, Carditis, Acute rheumatic fever, Enthesitis related arthritis, Juvenile

\section{Background}

Reactive arthritidesare inflammatory arthropathies where joint and extra articular manifestations follow an extra articular infection with arthritogenic bacteriaelike Chlamydia, Shigella and Campylobacter [1]. With frequent human leukocyte antigen (HLA) B27 positivity, they present as lower limb, asymmetrical oligoarthritis. Group A beta hemolytic streptococcal (GAS) infection of the throat causes several immune mediated diseases like acute rheumatic fever (ARF) and post streptococcal reactive arthritis (PSRA). Although considered part of the same spectrum, they differ in presentation, immunology, therapy and prognosis, making differentiation necessary [2].

Manuscript received: $5^{\text {th }}$ November 2017

Reviewed: $14^{\text {th }}$ November 2017

Author Corrected: $20^{\text {th }}$ November 2017

Accepted for Publication: 27 ${ }^{\text {th }}$ November 2017
Case 1: A 15-year-old boy with no previous personal or family history of arthritis presented with bilateral knee and anklearthritis with severe low spine pain, preventing him from standing or walking. He had high fever, chills and rigors but no antecedent respiratory or gastrointestinal infections. Investigations showed normal leucocyte count, elevated erythrocyte sedimentation rate (ESR) (120mm after one hour), positive $\mathrm{C}$ - reactive protein (CRP) and thrombo cytosis $\left(420000 / \mathrm{mm}^{3}\right)$. Antistreptolysin O (ASO) titre was elevated (500IU/1) but throat swab culture was sterile. Electrocardiogram (ECG) and echocardiography (ECHO) were normal. Anti nuclear antibody (ANA) and rheumatoid factor (RF) were negative. Possibilities of ARF, PSRA and seronegative spondyloarthropathy were considered.Aspirin with 


\section{Case Report}

antibiotics proved futile while naproxen for 2 weeks elicited partial response. Oral prednisolone elicited dramatic response and was tapered, with reintroduction of aspirin for 14 weeks with penicillin prophylaxis. Child then received naproxen for mild arthralgia and plantar fasciitis. A mild relapse six months later responded to steroids. He was noted to be HLA B27 positive. Repeat ECHO scan was normal but sacro-ilitis was evident. A diagnosis of enthesitis related arthritis was made. His eye examination was normal. Naproxen was restarted and could later be stopped with resumption of good quality of life for the past 2.5 years of follow up.

Case 2: A 9 year old boy suffered sore throat, followed three days later by severe and debilitating arthritis of right and left knees sequentially without fever. He responded to antibiotics and anti inflammatory drugs given by his doctor. Two months later, he had recurrence of bilateral knee joint arthritis three days following pharyngitis with elevated CRP (29mg/dl) and ASO (600IU/l) without carditis. He was treated for acute rheumatic fever, responded and put on oral penicillin prophylaxis, compliance to which was poor. Two and five months after the first recurrence, child suffered similar manifestations, each time after 4-5 days of a sore throat. Several months after his fourth episode, he presented in an asymptomatic state to us for evaluation, when normal ESR, CRP and ASO and throat swab culture were noted. But echocardiography revealed mitral valvulitis. On retrospective review of his records, a diagnosis of post streptococcal reactive arthritis seemed more likely, with late development of carditis, unlike the acute phase carditis noted in acute rheumatic fever. Child was continued on regular penicillin prophylaxisand followed up for 2 years when he had a mild relapse but responded well and then remained healthy.

\section{Discussion}

Acute rheumatic fever is conventionally diagnosed using modified Jones criteria [3] while Gewtiz et al proposed modifications emphasizing ECHO input [4]. PSRA was suspected in those not fulfilling these criteria after streptococcal infection.

Table-1: Diagnostic criteria [5] for PSRA.

A. Characteristics of the arthritis

1. Acute-onset arthritis, symmetrical or asymmetrical, usually non-migratory, can affect any joint

2. Persistent or recurrent

3. Poorly responsive to aspirin or non steroidal anti-inflammatory drugs

B. Evidence of antecedent group A streptococcal infection

C. Does not fulfill the modified Jones criteria for the diagnosis of acute rheumatic fever

PSRA vs ARF:

Table- 2: Differentiating points between acute rheumatic fever and post streptococcal reactive arthritis.

\begin{tabular}{|c|c|c|}
\hline Characteristic & Acute rheumatic fever & Post streptococcal reactive arthritis \\
\hline Age & $5-15$ years & Bimodal: 8-14 years and 21-37 years \\
\hline Latency period after streptococcal infection & $2-3$ weeks & 7-10 days \\
\hline Migratory arthritis & Yes & No \\
\hline Involved joints & Usually large joints only & Large, small and axial joints \\
\hline Joint involvement & Transient & Persistent or recurrent \\
\hline Carditis frequency & Occurs in about $60 \%$ & Occurs in about 6\% \\
\hline Carditis onset & During acute phase & Several months later, if occurs \\
\hline HLA B27 positivity & None & Some patients with axial involvement \\
\hline Genetic markers & DRB1*01 & DRB1*16 \\
\hline $\begin{array}{c}\text { Acute phase reactants elevation } \\
\text { Positive evidence of preceding streptococcal } \\
\text { infection }\end{array}$ & Marked & Moderate \\
\hline Response to aspirin & $30 \%$ & Not dramatic \\
\hline $\begin{array}{c}\text { Erythema nodosum or erythema } \\
\text { multiforme[6] }\end{array}$ & Sporadic occurrence & Noted more frequently \\
\hline
\end{tabular}


Case Report

Secondary prophylaxis guidelines provided by the World Health Organization (WHO) for ARF may be followed in children who had satisfied modified Jones criteria in their acute phase with overlapping features of ARF and PSRA (case 1) or those with PSRA who develop carditis subsequently (case 2). Uncertainty exists regarding prophylaxis with American Heart Association advocating stopping it after one year in well patients without carditis in acute phase [7]. Presence of non group A streptococcal infections, as indicated by higher ratios of ASO titer to anti DNAase B titer have been associated with lesser incidence of carditis and so recommended to decide about prophylaxis but are yet to be validated[8].

Challenges in the presented cases: The first child with polyarthritis (one major manifestation), fever and elevated acute phase reactants (two minor manifestations) would qualify for the diagnosis of acute rheumatic fever. However, presence of axial skeletal involvement, non-migratory persistent arthritis, absence of carditis, normal leukocyte count and lack of dramatic response to aspirin favour the diagnosis of PSRA rather than ARF. Owing to the risk of carditis following a possible relapse, it is preferable to contniue penicillin prophylaxis for 5 years as per WHO guidelines. Polytendinitis, tenosynovitis and enthesitis have been documented in PSRA and so were not contradictory to the initial consideration [9]. However, on follow up, owing to a chronic lingering course and enthesitis in this adolescent boy with subsequent HLA B27 positivity and sacro-ilitis, revision of diagnosis to enthesitis related arthritis was made and child treated for the same currently.

The second child whose diagnosis was delayed, developed carditis seven months after the acute episode. This has been noted to occur in about 5.8\% of PSRA patients[10]. With appropriate anti-inflammatory drugs, arthralgia completely resolved in this child. On a compliant oral penicillin prophylaxis, he didn't experience a relapse for the past 1.5 years. Children who develop recurrent break through arthritis despite oral penicillin prophylaxis should be considered for intramuscular injections of benzathine penicillin, as recurrence rates are less[11], but couldn't be adopted in this child due to consent refusal. Prophylaxis in this child must be continued until 25 years of age.

\section{Conclusion}

Post streptococcal reactive arthritis presents a diagnostic challenge, especially in its differentiation from acute rheumatic fever. The most important factors that would help in deciding about the antibiotic prophylaxis would be the presence or absence of co-existent carditis and fulfillment of modified Jones criteria in the acute phase of illness. In those with carditis and in those who have features overlapping with ARF, it is preferable to follow the WHO guidelines for antibiotic prophylaxis for ARF patients until convincing data on this aspect are available. Long term follow up is essential to monitor evolving pattern of joint diseases.

Authors' contributions: All authors contributed equally in terms of patient management, follow up and data collection. AS made literature search and prepared the primary manuscript. MJ and BS proof read and edited it. KSR helped with literature search.

Conflicts of interest: The authors declare they have no conflicts of interest.

Funding: No funding was provided for this work. The said patients received free hospital care in Saveetha Medical College and Hospital as per the hospital's routine policy
Ethics approval: The article including the case descriptions has been approved by the ethics committee of Saveetha Medical College and Hospital.

\begin{abstract}
Abbreviations
PSRA: Post streptococcal reactive arthritis, ARF: Acute rheumatic fever, HLA: Human leucocyte antigen, GAS: Group A beta hemolytic streptococcus, ESR: Erythrocyte sedimentation rate, CRP: C reactive protein, ASO: Anti streptolysin O, ECG: Electrocardiogram, ECHO: Echo-cardiography, ANA: Anti nuclear antibody, RF: Rheumatoid factor, WHO: World health organization, DNAase: Deoxyribonuclease
\end{abstract}

\section{References}

1. Cassidy JT, Petty RE, Laxer RM, Lindsley CB. Textbook of Pediatric Rheumatology. Sixth edition. Philadelphia. Saunders Elsevier;2011

2. Uziel Y, Perl L, Barash J, Hashkes PL. Poststreptococcal reactive arthritis in children: a distinct entity from acute rheumatic fever. Pediatric Rheumatology2011;9:32DOI:10.1186/1546-0096-9-32. 
Case Report

3. WHO. Rheumatic fever and rheumatic heart disease. Report of a WHO expert consultation. Geneva, 29 October-1 November 2001. WHO Technical Report Series. 2004; 923:1. url:http:// www. who. int/iris/handle/10665/42898

4. Gewitz MH, Baltimore RS et al. Revision of the Jones criteria for the diagnosis of Acute rheumatic fever in the era of echocardiography. Circulation 2015; 131. doi: 10. 1161/CIR.0000000000000205. Epub 2015 Apr 23.

5. Ayoub EM, Ahmed S: Update on complications of group A streptococcal infections. Curr Probl Pediatr 1997;27(3):90-101.DOI: http://dx. doi. org/ 10. 1016/S0045-9380(97)80010-2.

6. Jansen TLThA, Janssen M, Van Riel PLCM. Acute rheumatic fever or post-streptococcal reactive arthritis: a clinical problem revisited. British Journal of Rheumatology 1998: 37:335-340.

7. Gerber MA, Baltimore RS, Eaton CB, Gewitz M, Rowley AH, Shulman ST,et al. Prevention of rheumatic fever and diagnosis and treatment of acute Streptococcal pharyngitis: a scientific statement from the American Heart Association Rheumatic Fever, Endocarditis, and Kawasaki Disease Committee of the Council on Cardiovascular Disease in the Young, the Interdisciplinary Council on Functional Genomics and Translational Biology, and the Interdisciplinary Council on Quality of Care and Outcomes Research: endorsed by the American Academy of Pediatrics. Circulation 2009; 119(11):1541-51. DOI:10.1161/ CIRCULATIONAHA.109.191959

8. Jansen TLThA, Janssen M, Traksel R, de Jong AJL. A clinical and serological comparison of group A versus non-group A streptococcal reactive arthritis and throat culture negative cases of poststreptococcal reactive arthritis. Ann Rheum Dis $1999 ; 58: 410-414$

9. Sarakbi HA, Hammoudeh $\mathrm{M}$ et al. Post streptococcal reactive arthritis and the association with tendonitis, tenosynovitis and enthesitis. J Clin Rheumatol 2010; 16:3-6. doi: 10.1097/RHU. 0b013e $3181 \mathrm{c} 3444 \mathrm{c}$

10. Ahmed S, Ayoub EM, Scornik JC, Wang CY, She JX. Poststreptococcal reactive arthritis: clinical characteristics and association with HLA-DR alleles. Arthritis Rheum 1998, 41(6):1096-102. DOI:10.1002/1529-0131(199806)41: 6<1096:: AIDART17>3.0.CO;2-Y

11. Manyemba J, Mayosi BM. Penicillin for secondary prevention of rheumatic fever. Cochrane Database Syst Rev. 2002; (3): CD002227. DOI:10. 1002/14651858.CD002227

\section{How to cite this article?}

Swaminathan A, Muhammad N, Benjamin S, Rajagopalan K.S. Post streptococcal reactive arthritis in children: varied presentations, differentiation from acute rheumatic fever and antibiotic prophylaxis. Int J Pediatr Res. 2017;4(11):694-697.doi:10. 17511/ijpr.2017.i11.10. 